\title{
DOI : 10.5846/stxb201303210477
}

刘霞, 胡海清,李为海, 孙程坤, 黄超,赵希宽, 孙龙.寒温带岛状林沼泽土壤呼吸速率和季节变化.生态学报,2014,34(24):7356-7364.

Liu X, Hu H Q, Li W H, Sun C K, Huang C, Zhao X K, Sun L. Soil respiration rate and its seasonal variation of island forested swamp in cool temperate zone.Acta Ecologica Sinica, 2014,34(24):7356-7364.

\section{寒温带岛状林沼泽土壤呼吸速率和季节变化}

\author{
刘 霞 ${ }^{1,2}$, 胡海清 ${ }^{1, *}$, 李为海 ${ }^{2}$, 孙程坤 $^{3}$, 黄 超 ${ }^{2}$, 赵希宽 ${ }^{2}$, 孙 龙 $^{1}$ \\ (1. 东北林业大学林学院, 哈尔滨 $150040 ; 2$.大兴安岭地区农业林业科学研究院 南瓮河嫩江源生态站, 加格达奇 165000 ; \\ 3. 大兴安岭地区营林局, 加格达奇 165000)
}

\begin{abstract}
摘要:2011 年生长季内利用静态箱-气相色谱法, 研究了寒温带典型湿地白桦 (Betula platyphylla) 岛状林沼泽、兴安落叶松 (Larix gmelinii) 岛状林沼泽土壤呼吸速率的季节动态及其主要环境因子, 利用壕沟隔断法对土壤呼吸各组分间的差异进行研 究。结果表明:生长季白桦和兴安落叶松岛状林沼泽土壤呼吸速率具有明显的季节性规律, 土壤呼吸总速率分别为 368.60 和 $312.46 \mathrm{mg} \mathrm{m}^{-2} \mathrm{~h}^{-1}$, 异养呼吸速率分别为 300.57 和 $215.70 \mathrm{mg} \mathrm{m}^{-2} \mathrm{~h}^{-1}$, 占土壤呼吸总速率的 $81.5 \%$ 和 $69.0 \%$; 自养呼吸速率为 68.03 和 $96.76 \mathrm{mg} \mathrm{m}^{-2} \mathrm{~h}^{-1}$, 占土壤呼吸总速率的 $18.5 \%$ 和 $31.0 \%$ 。不同处理条件下的土壤呼吸在季节变化上表现基本一致,高 峰期都发生在夏季; 土壤呼吸与温度呈极显著相关性,但与土壤湿度的相关性较差。生长季白桦和兴安落叶松岛状林沼泽土壤 呼吸总量分别为 12.64 和 $10.61 \mathrm{t} / \mathrm{hm}^{2}$ 。
\end{abstract}

关键词: 土壤呼吸速率; 季节变化规律; 岛状林沼泽; 大兴安岭

\section{Soil respiration rate and its seasonal variation of island forested swamp in cool temperate zone}

\author{
LIU Xia ${ }^{1,2}$, HU Haiqing ${ }^{1, *}$, LI Weihai ${ }^{2}$, SUN Chengkun ${ }^{3}$, HUANG Chao ${ }^{2}$, ZHAO Xikuan ${ }^{2}$, SUN Long ${ }^{1}$ \\ 1 College of Forestry, Northeast Forestry University, Harbin 150040, China \\ 2 Forestry and Agriculture of Academy in the DaXing'an Mountains. Nenjiangyuan Forest ecosystem research station, Jiagedaqi 165000, China \\ 3 Bureau of Forestry in the DaXing' an Mountains, Jiagedaqi 165000, China
}

\begin{abstract}
Wetlands play a critical role in the global carbon cycle, wetland soil carbon stock accounting for about one-third of the global carbon storage, especially in the high latitude regions. DaXing' An Mountains region is located in an area of high latitude which is a major important area for global climate change. It has a cold and dry climate, small amount of soil microbes and low soil respiration rate. It is China's biggest warning area of global warming. Hence it is important to research on soil respiration and its relation to environmental factors in different types of wetlands in DaXing' An Mountains region. The seasonal dynamics of soil respiration were measured, respectively, in boreal typical wetlands birch (Betula platyphylla) island forest swamp and larch (Larix gmelinii) island forest marsh, using a static tank-gas chromatography method in the 2011 growing season. The Trenches Partition methosed was used to study the differences between the components of soil respiration. Our results showed that: Birch and larch island forest swamp soil respiration rate had obvious seasonal law in the growing season. Total soil respiration rates were 368.60 and $312.46 \mathrm{mg} \mathrm{m}^{-2} \mathrm{~h}^{-1}$, heterotrophic respiration rates were 300.57 and $215.70 \mathrm{mg} \mathrm{m}^{-2} \mathrm{~h}^{-1}$, autotrophic respiration rate were 68.03 and $96.76 \mathrm{mg} \mathrm{m}^{-2} \mathrm{~h}^{-1}$. The peak period of soil respiration rates occurred during summer. Soil respiration was significantly correlated with temperature, while soil respiration was not significantly correlated with soil humidity. The soil temperatures rates of birch swamp were higher than the larch
\end{abstract}

基金项目:国家自然科学基金项目(31070544); 国家公益性行业科研专项(201004074)

收稿日期:2013-03-21; 网络出版日期:2014-03- 19

* 通讯作者 Corresponding author.E-mail: huhq-cf@ nefu.edu.cn 
swamps, soil respiration rates of birch swamp were significantly correlated with soil temperature of 0 - 40 $\mathrm{cm}$ soil layer, which indicated the large space extent of microbial activity in this forest type; while soil respiration rates of larch swamp was significantly correlated with soil temperature of 5-15 cm soil layer, which indicated the small space extent of microbial activity and low contribution rate in this forest type. Soil respiration rate was $12.64 \mathrm{t} / \mathrm{hm}^{2}$, heterotrophic respiration and autotrophic rates respiration were accounted for $81.5 \%$ and $18.5 \%$ of the total Soil respiration rate in birch swamp. Soil respiration rates was $10.61 \mathrm{t} / \mathrm{hm}^{2}$, heterotrophic respiration and autotrophic respiration rates were accounted for $69.0 \%$ and $31.0 \%$ of the total soil respiration rates in larch swamps during the growing season. The soil respiration rate of soil microbes was significant higher than the soil respiration rate of root system in both forest types. The heterotrophic respiration ( $2.57 \mathrm{t} /$ $\left.\mathrm{hm}^{2}\right)$ of birch swamps was higher than that of larch swamp, while autotrophic respiration $\left(0.54 \mathrm{t} / \mathrm{hm}^{2}\right)$ of birch swamps was lower than that of larch swamp when the soil respiration rate of birch swamps was larger than $2.03 \mathrm{t} / \mathrm{hm}^{2}$ of birch swamps. The autotrophic respiration rate of microbes was in dominant position and accounted for $68.0 \%-84.4 \%$ of total soil respiration, while the heterotrophic respiration of root systems accounted for $15.6 \%-32.0 \%$ of total soil respiration. The soil respiration rate was significantly correlated with soil SOC in the case of relatively stable temperature and soil moisture, the soil respiration rate increased with SOC. The biomass, coverage of herbaceous plants was consistent wit autotrophic respiration rate. The soil respiration rate was large when the large biomass and coverage.

Key Words : soil respiration rate; seasonal variation; island forested swamps; DaXing'An Mountains

全球变暖是人类目前面临的主要环境问题之 一, $\mathrm{CO}_{2}$ 是大气中重要的温室气体, 它对全球变暖贡 献率达 $60 \%$ 以上 $^{[1]}$ 。全球每年通过土壤呼吸向大气 释放的碳约为 $68 \mathrm{Pg}$, 仅次于全球总初级生产力, 超 过全球陆地生态系统的净初级生产力, 是化石燃料 燃烧释放的碳量的十多倍 ${ }^{[2]}$ 。同时土壤呼吸又是陆 地生态系统碳素循环的主要环节,并已成为向大气 释放 $\mathrm{CO}_{2}$ 最大的源 ${ }^{[1,3]}$, 全球森林土壤碳库是森林植 被碳库的 2 倍多 ${ }^{[4]}$, 且森林土壤 $\mathrm{CO}_{2}$ 通量占森林生 态系统呼吸总量的 $40 \%-80 \%{ }^{[5]}$, 所以, 土壤呼吸即 使发生微小的变化, 对全球碳循环, 特别是对大气 $\mathrm{CO}_{2}$ 浓度的变化将产生重大影响。

土壤呼吸 $\left(R_{S}\right)$ 作用, 严格意义上讲是指未受扰 动的土壤中产生 $\mathrm{CO}_{2}$ 的所有代谢作用 ${ }^{[6]}$, 包括 3 个 生物学过程 (植物根呼吸、土壤微生物呼吸及土壤动 物呼吸) 和一个非生物学过程 (含碳物质的化学氧化 作用)。一般认为, 土壤呼吸作用主要来自于土壤微 生物对有机质 (土壤有机质、枯枝落叶和死根等) 的 分解 (异养呼吸作用, $R_{H}$ ) 及植物根系呼吸 (自养呼 吸作用, $\left.R_{A}\right)$ 两大部分。研究表明,土壤呼吸释放的 $\mathrm{CO}_{2}$ 约 30\%-50\%来自根系活动或自氧呼吸作用, 其 余部分主要来源于土壤微生物对有机质的分解 作用 ${ }^{[7]}$ 。

温度是影响土壤呼吸的关键因素, 两者的相关
性较好。相关研究表明, 湿地土壤呼吸速率夏季最 大, 春秋季次之, 冬季最低 ${ }^{[8-11]}$ 。而水位是控制湿地 碳平衡在空间和时间分布上的重要因子之一 ${ }^{[12]}$; 土 壤含水量对呼吸速率的影响较为复杂, 且不如温度 明显, 土壤含水量太高 (高于土壤最大持水量的 $66.3 \%$ ) 或太低(低于土壤体积的 5\%-20\%) 将导致 土壤呼吸速率降低或停止 ${ }^{[13]}$, 湿地土壤呼吸速率随 着水位的降低而增加 ${ }^{[14]}$, 呈现明显的空间差异, 但 在水分条件不能成为限制因子时温度对其具有显著 的影响 $^{[9]}$ 。因此, 有必要加强不同区域间和不同湿 地类型的对比研究。

弄清土壤呼吸各个组分在整个土壤呼吸中的贡 献, 才能更好地研究土壤碳获取, 而且在了解生态系 统碳收支状况时也需要区分土壤呼吸各组分 ${ }^{[15]}$; 同 时它对于研究全球碳循环、全球变暖及森林植物生 理生态和土壤生态都具有非常重要的意义 ${ }^{[15-16]}$ 。

\section{1 研究区概况与研究方法}

\section{1 研究区概况}

研究地点位于黑龙江省大兴安岭地区农林科学 院的嫩江源森林-湿地生态系统定位站,该站位于大 兴安岭林区东南部, 伊勒呼里山南麓, 松岭区境内, 北以伊勒呼里山脉为界, 东至二根河, 南以松岭区同 加格达奇林业局界为准。地理坐标为北纬 $51^{\circ} 05^{\prime}$ 
$07^{\prime \prime}-51^{\circ} 39^{\prime} 24^{\prime \prime}$, 东经 $125^{\circ} 07^{\prime} 55^{\prime \prime}-125^{\circ} 50^{\prime} 05^{\prime \prime}$ 。该区 总面积为 $229523 \mathrm{hm}^{2}$, 全部为国有林地。海拔高度 为 500-800 m, 属低山丘陵地带, 河谷宽阔, 气候属 于寒温带大陆性季风气候, 年平均气温 $-3^{\circ} \mathrm{C}$, 极端最 低温 $-48{ }^{\circ} \mathrm{C}$, 年日照时数 $2500 \mathrm{~h}$, 无霜期 $90-100 \mathrm{~d}$, 植物生长期为 $110 \mathrm{~d}$, 年降雨量 $500 \mathrm{~mm}$ 。地带性土 壤为棕色针叶林土。

岛状林沼泽湿地形成在河流中下游比较开阔的 河谷地带, 由于微地形变化, 森林成岛屿状分布在大 面积草丛沼泽湿地中, 故称岛状沼泽林。本实验重 点是研究两种典型的湿地岛状林沼泽, 以优势树种
进行分类, 分别为: 兴安落叶松 (Larix gmelini)-岛状 林沼泽和白桦 (Betula platyphylla) - 岛状林沼泽, 实验 样地理化性质, 样地生物多样性、生物量及其草本盖 度数据介绍 ( $1 \mathrm{~m} \times 1 \mathrm{~m}, 5$ 个重复) (表 1$)$ 。两样地相 隔 $50 \mathrm{~m}$, 由栈道相连。岛状林沼泽的水位都常年处 于地表以下, 只有融冻期或雨季时,才会有短期斑 块状积水,而落叶松地表几乎从无积水。实验样地 5 月末植物开始萌发, $7 、 8$ 月份植物生长旺盛, 9 月植 物开始枯黄、树叶调落, 10 月份偶有降雪, 地表清晨 出现薄冰层; 因此实验季节划分为 $5 、 6$ 月份为研究 的春季, $7 、 8$ 月份为生长的夏季, $9 、 10$ 月份为秋季。

\section{表 1 森林沼泽类型及其基本特征}

Table 1 Types and characteristics of forested swamps

\begin{tabular}{|c|c|c|c|c|c|c|}
\hline $\begin{array}{l}\text { 沼泽类型 } \\
\text { Swamp types }\end{array}$ & $\begin{array}{l}\text { 乔木 } \\
\text { Trees }\end{array}$ & $\begin{array}{l}\text { 灌木 } \\
\text { Shrubs }\end{array}$ & $\begin{array}{l}\text { 草本 } \\
\text { Herbs }\end{array}$ & $\begin{array}{l}\text { 草本盖度/\% } \\
\text { Herbs coverage }\end{array}$ & $\begin{array}{c}\text { 草本生物量 } \\
\text { Aboveground } \\
\text { biomass of herbs/ } \\
\left(\mathrm{g} / \mathrm{m}^{2}\right)\end{array}$ & $\begin{array}{c}\text { 土壤有机碳 } \\
\text { Organic carbon } \\
\text { of soil/ } \\
(\mathrm{g} / \mathrm{kg})\end{array}$ \\
\hline $\begin{array}{l}\text { 兴安落叶松-岛状林沼泽 } \\
\text { Larix gmelinii swamp }\end{array}$ & 1 & 3,4 & $6,7,8,9$ & $44 \%$ & 177.70 & 91.00 \\
\hline $\begin{array}{l}\text { 白桦-岛状林沼泽 } \\
\text { Betula platyphylla swamp }\end{array}$ & 2,1 & 5 & $7,8,9,10,11$ & $63 \%$ & 327.08 & 125.91 \\
\hline
\end{tabular}

1: 落叶松 Larix gmelinii 2: 白桦 Betula platyphylla 3: 柳叶绣线菊 Spiraea salicifolia 4: 山刺玫 Rosa davurica 5: 笃斯越桔 Vaccinium uliginosum 6: 蚊子草 Filipendula palmata 7: 鹿蹄草 Pyrola incarnate 8: 小叶樟 Calamagrostis angustifolia 9: 苔草 Moss 10: 小白花地榆 Sanguisorba parviflora 11 : 大花马先蒿 Pedicularis grandiflora

\section{2 研究方法}

\subsection{1 气体采集与分析}

土壤呼吸速率 $\left(R_{S}\right)$ 的采集使用静态暗箱法。春 季取样前将规格为 $50 \mathrm{~cm} \times 50 \mathrm{~cm} \times 10 \mathrm{~cm}$ 不锈钢底座 插人土壤中 $10 \mathrm{~cm}$ 固定, 底座上部四周带有凹槽, 取 样时注水密封。整个生长季底座放在试验地不动, 以保证对底座内部植被和土壤的干扰最小。不锈钢 顶箱规格为 $50 \mathrm{~cm} \times 50 \mathrm{~cm} \times 50 \mathrm{~cm}$, 箱内顶部安有直径 $10 \mathrm{~cm}$ 风扇, 取样时风扇保持转动, 避免箱内出现气 体浓度差, 箱顶部直径 $1 \mathrm{~cm}$ 内置橡胶塞作为取样口, 箱侧面 2 个小孔用于数字温度计探头和风扇电源线 通过, 分别用橡胶塞和硅胶密封。顶箱外都粘贴保 温材料, 以减少箱内温度波动。每个类型沼泽内设 置 3 次重复。

土壤异养呼吸 $\left(R_{H}\right)$ 和根系自养呼吸 $\left(R_{A}\right)$ 采用 根去除挖壕法 ${ }^{[17]}$ 间接测定。2011 年 4 月下旬在每 块固定样地的外围处随机选择 3 个 $60 \mathrm{~cm} \times 60 \mathrm{~cm}$ 小 样方，在小样方四周挖壕深至 40-60 cm（植物根 系分布层以下), 壕内用双层厚塑料布隔离小样方
周围的根系; 再除去小样方内所有活体植物, 并在 随后的测定中始终保持小样方内没有活体植物; 最 后每个小样方内安置一个 $50 \mathrm{~cm} \times 50 \mathrm{~cm} \times 10 \mathrm{~cm}$ 不锈 钢底座, 安置方法前同。挖好后 2 个月 (2011 年 6 月 上旬)，与常规的 $R_{S}$ 测定同步,每 $10 \mathrm{~d}$ 测定 1 次挖壕 样方内的土壤呼吸速率及其相关的环境因子, 测定 方法与前同。这样, 挖壕样方内的土壤呼吸速率即 为 $R_{H}$; 挖壕样方与非挖壕样方的土壤呼吸速率之差 即为 $R_{A}$ 。上述测定基本涵盖了本地区的土壤温度和 含水量的季节变化范围。

取样时间为 2011 年的 6 月 5 日至 10 月 15 日， 取样频率为每月 3 次,约 $10 \mathrm{~d}$ 取 1 次, 每次取样在 9:00-11:00 完成。用 $60 \mathrm{~mL}$ 聚氯乙烯医用注射器 经三通阀连接铁针头通过箱顶部橡胶塞取样。取样 时,每个静态箱在 $30 \mathrm{~min}$ 内取 4 管气体, 分别在静态 箱封闭后的 $0 、 10 、 20$ 和 $30 \mathrm{~min}$ 时进行。气体样品用 注射器取出后转移进 $500 \mathrm{~mL}$ 的铝塑复合气袋 (大连 光明化工厂生产) 储存。带回试验室在 1 周内用气 相色谱仪 (HP5890 II) 测定 $\mathrm{CO}_{2}$ 浓度。利用以下公 
式计算土壤呼吸速率:

$$
F=\frac{\mathrm{d} c}{\mathrm{~d} t} \frac{M}{V_{0}} \frac{P}{P_{0}} \frac{T_{0}}{T} H^{[18]}
$$

式中, $F$ 为气体呼吸速率 $\left(\mathrm{mg} \mathrm{m}^{-2} \mathrm{~h}^{-1}\right), \mathrm{d} c / \mathrm{d} t$ 为采样 时气体浓度随时间变化的直线斜率, $M$ 为被测气体 的摩尔质量, $P$ 为采样点的大气压, $V_{0} 、 P_{0} 、 T_{0}$ 分别为 标准状态下的气体摩尔体积、标准大气压和绝对温 度, $H$ 为采样箱的高度。根据生长季 $(6-10$ 月) 每 月上、中、下旬 $\mathrm{CO}_{2}$ 排放通量的实测数据, 依此公式 计算的通量结果, 求其生长季所测各数值平均值为 生长季节土壤呼吸平均速率, 并计算各月份的排放 量加和得到土壤呼吸在生长季的呼吸总量。

\subsection{2 环境因子测定}

土壤呼吸速率采集的同时,使用 JM624 便携式 数字温度计原位同步测定气温、箱温、 $0 、 5 、 10 、 15$ 、 $20 、 30$ 和 $40 \mathrm{~cm}$ 土壤温度, 温度值为试验过程中每次 分别在实验开始时, 实验中, 实验结束前监测 3 次温 度, 取其平均值。沼泽水深通过在采样箱附近挖井, 用固定直尺直接测量。2011 年生长季春季多雨, 6 月中旬到下旬土壤含水率较高, 夏季少雨较干旱, 除 6 月中旬可观测到水位 $-22.8--37.5 \mathrm{~cm}$, 其他时期 无水位。

\subsection{3 数据统计分析}

用 SPSS 13.0 统计分析软件包做环境因素与排 放通量的回归方程及相关性分析, 并用 Microsoft Office Excel 2003 对数据进行分析处理。

\section{2 结果与分析}

\section{1 生长季内环境因子变化}

整个生长季降水量都较低, 只有春季 $6 、 7$ 月出 现较大降水, 加之土壤融冻过程, 使土壤含水率较 高,但水位也低于 $-20 \mathrm{~cm}, 7$ 月份降水主要集中在上 旬,并且从 7 月中旬开始观测期内水位都低于 -40 $\mathrm{cm}$, 并于 9 月中下旬观测日出现降雪, 10 月份无降 水过程(图 1)。生长季白桦岛状林沼泽土壤 $15 \mathrm{~cm}$ 深度土壤含水率平均值 $35.37 \%$, 落叶松岛状林沼泽 土壤含水率平均值 $24.23 \%$ 。

生长季 2 个样地温度波动曲线规律基本一致 （图 2), 大气、箱内、地表 $0 \mathrm{~cm}$ 温度春、夏季都保持较 高的波动范围,8 月末开始显著下降,而地表深层土 壤温度略滞后于表层温度, 夏季达到生长季最高温
度值。生长季白桦岛状林沼泽各土壤层温度平均值 均高于同层落叶松岛状林沼泽。

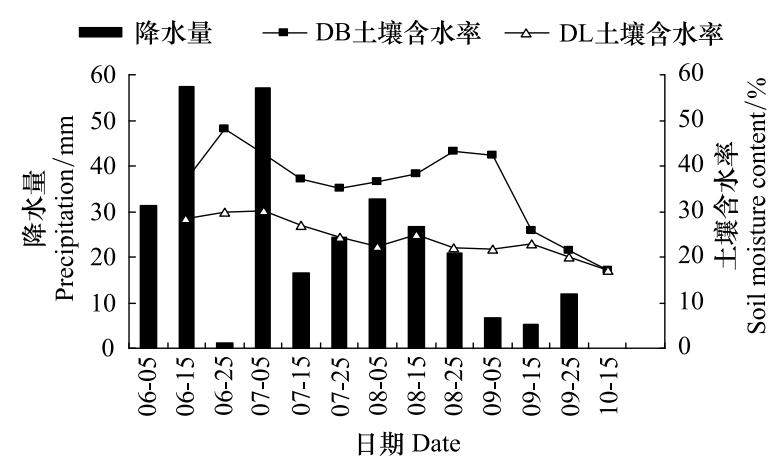

图 1 嫩江源地区 2011 年降水量、土壤含水率季节变化

Fig.1 Monthly variations of the rain precipitation and soil moisture content in 2011 year in Nen Jiangyuan
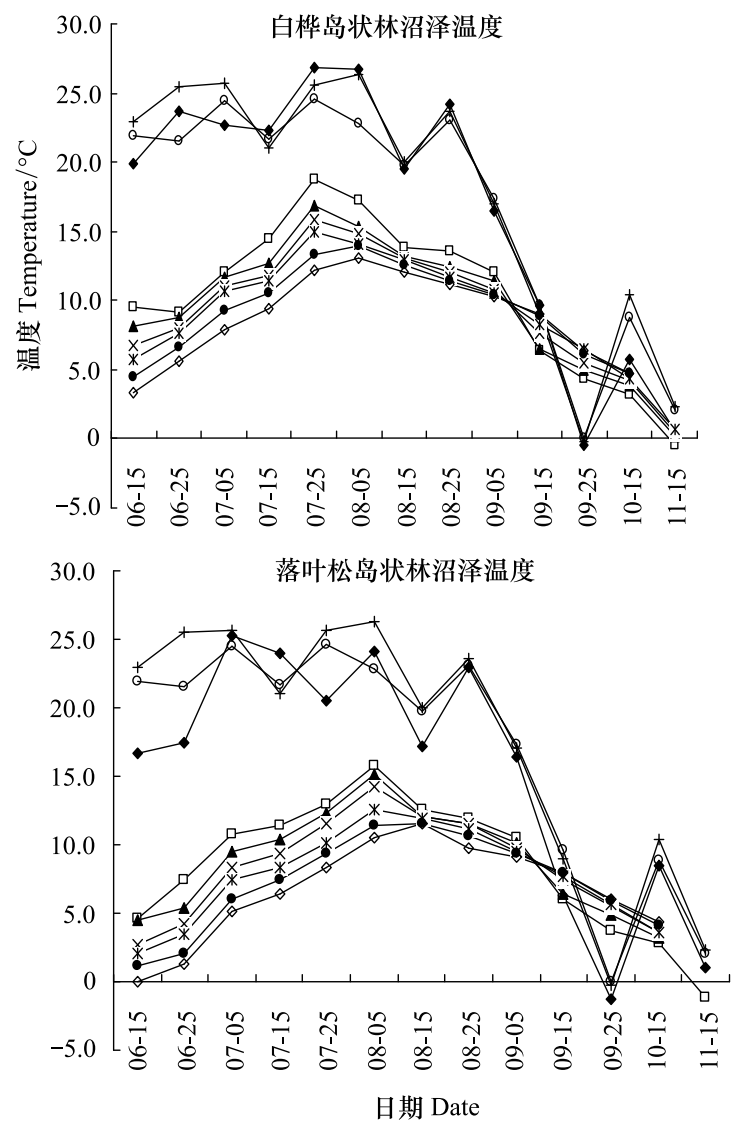

图 2 不同沼泽类型温度动态

Fig.2 Dynamics of temperature from different swamps style

2.2 土壤呼吸速率的季节动态

白桦岛状林沼泽土壤呼吸 $\left(R_{S}\right)$ 速率具有明显的 季节变化规律 (图 3), 生长季表现为单峰型曲线, 平 均速率为 $368.60 \mathrm{mg} \mathrm{m}^{-2} \mathrm{~h}^{-1}$, 波动范围为 121.52 $638.23 \mathrm{mg} \mathrm{m}^{-2} \mathrm{~h}^{-1}, 8$ 月中旬达到峰值。6 月中旬开 
始土壤呼吸速率呈逐渐升高的趋势, 7 月中旬一 8 月 末表现为土壤呼吸速率波动性高峰区域 (平均速率 $562.74 \mathrm{mg} \mathrm{m}^{-2} \mathrm{~h}^{-1}$, 较季节平均速率提高了 $65.5 \%$ )。 之后逐渐下降, 直至 10 月末, 保持较低的呼吸值。 土壤异养呼吸 $\left(R_{H}\right)$ 季节变化规律与 $R_{S}$ 较一致, 呼吸 速率峰值出现在 8 月上旬, 波动范围为 $61.03-$ $601.46 \mathrm{mg} \mathrm{m}^{-2} \mathrm{~h}^{-1}$, 平均速率为 $300.57 \mathrm{mg} \mathrm{m}^{-2} \mathrm{~h}^{-1}$ 。 由此可得根系自养呼吸 $\left(R_{A}\right)$ 平均速率为 $68.03 \mathrm{mg}$ $\mathrm{m}^{-2} \mathrm{~h}^{-1}$ 。

兴安落叶松岛状林沼泽土壤呼吸 $\left(R_{S}\right)$ 排放具有 明显的季节变化规律,生长季表现为双峰型曲线, 平

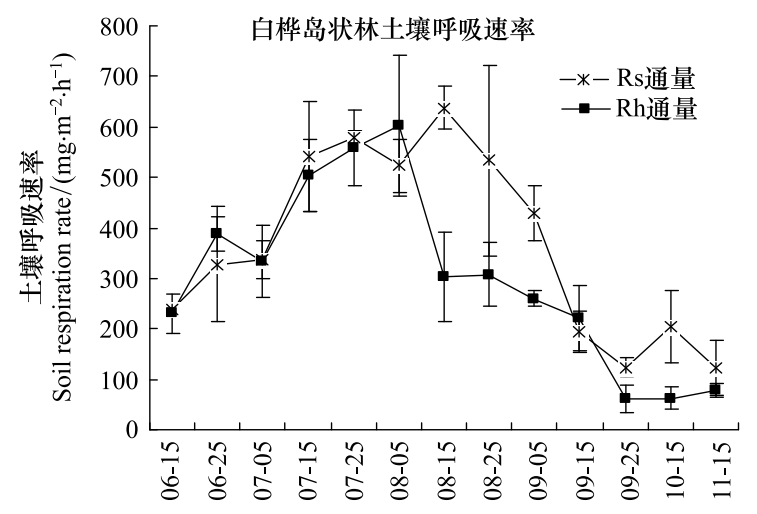

图 3 岛状林沼泽土壤呼吸速率的季节变化规律

Fig.3 Seasonal variations of soil respiration rate in island-forested swamps

温度对岛状林沼泽土壤呼吸的影响显著, 白桦 岛状林沼泽 $R_{S}$ 与 $R_{H}$ 排放与空气温度表现为显著地 相关性 $(P<0.05)$,与其他土壤层和箱内温度都表现 为极明显相关性; 落叶松岛状林沼泽 $R_{S}$ 排放与地表 $5 \mathrm{~cm} 、 10 \mathrm{~cm}$ 、箱内温度表现为极显著地相关性 $(P<$
均速率为 $312.46 \mathrm{mg} \mathrm{m}^{-2} \mathrm{~h}^{-1}$, 波动范围为 $79.96-$ $617.68 \mathrm{mg} \mathrm{m}^{-2} \mathrm{~h}^{-1}, 8$ 月上旬达到呼吸速率峰值, 6 月 中旬开始土壤呼吸速率逐渐升高, 7 月中旬一 9 月上 旬表现为土壤呼吸速率的波动性高峰区域 (平均速 率为 $420.94 \mathrm{mg} \mathrm{m}^{-2} \mathrm{~h}^{-1}$, 较季节平均速率提高了 $64.2 \%$ )。之后逐渐下降, 秋末又有小幅度回升, 出 现小高峰。土壤异养呼吸 $\left(R_{H}\right)$ 季节变化规律与 $R_{S}$ 较一致, 呼吸速率峰值出现在 8 月下旬 (601.46 mg $\mathrm{m}^{-2} \mathrm{~h}^{-1}$ ), 波动范围为 114.23 - $331.45 \mathrm{mg} \mathrm{m}^{-2} \mathrm{~h}^{-1}$, 平 均速率为 $215.70 \mathrm{mg} \mathrm{m}^{-2} \mathrm{~h}^{-1}$ 。由此可得根系自养呼 吸 $\left(R_{A}\right)$ 平均速率为 $96.76 \mathrm{mg} \mathrm{m}^{-2} \mathrm{~h}^{-1}$ 。

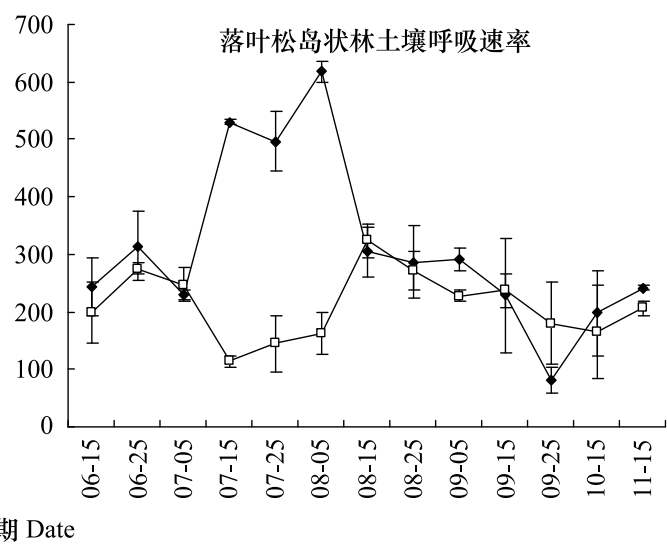

$0.01)$, 与土壤 $15 \mathrm{~cm}$ 、空气温度表现为显著相关性 $(P<0.05), R_{H}$ 排放与地表 $5 \mathrm{~cm} 、 10 \mathrm{~cm} 、 15 \mathrm{~cm}$ 、空气、 箱内温度表现为极显著地相关性 $(P<0.01)($ 表 2$)$ 。 白桦和落叶松岛状林沼泽土壤含水率与土壤呼吸相 关性分别为 $0.242 、 0.373$,均未达到显著水平。

表 2 土壤呼吸与温度的相关分析

Table 2 Correlation analysis of between soil respiration rate and temperature

\begin{tabular}{|c|c|c|c|}
\hline $\begin{array}{l}\text { 沼泽类型 } \\
\text { Swamp types }\end{array}$ & & $\begin{array}{c}\text { 回归分析 } \\
\text { Regression analyse }\end{array}$ & $R^{2}$ \\
\hline \multirow{6}{*}{$\begin{array}{l}\text { 白桦-岛状林沼泽 } \\
\text { Betula platyphylla swamp }\end{array}$} & $\ln \left(F_{\mathrm{Rs}}\right)=0.0533 \mathrm{~T}_{0}+4.878$ & $\ln \left(F_{\mathrm{Rs}}\right)=0.0955 \mathrm{~T}_{5}+4.783 \quad \ln \left(F_{\mathrm{Rs}}\right)=0.112 \mathrm{~T}_{10}+4.684$ & $R_{0}^{2}=0.777 \quad R_{5}^{2}=0.859 \quad R_{10}^{2}=0.860$ \\
\hline & $\ln \left(F_{\mathrm{Rs}}\right)=0.120 \mathrm{~T}_{15}+4.645$ & $\ln \left(F_{\mathrm{Rs}}\right)=0.125 \mathrm{~T}_{20}+4.619 \quad \ln \left(F_{\mathrm{Rs}}\right)=0.133 \mathrm{~T}_{30}+4.601$ & $R_{15}^{2}=0.843 \quad R_{20}^{2}=0.795 \quad R_{30}^{2}=0.678$ \\
\hline & $\ln \left(F_{\mathrm{Rs}}\right)=0.125 \mathrm{~T}_{40}+4.757$ & $\ln \left(F_{\mathrm{Rs}}\right)=0.0522 \mathrm{~T}_{\text {air }}+4.848$ & $R_{40}^{2}=0.568 \quad R_{\text {air }}^{2}=0.691$ \\
\hline & $\ln \left(F_{\mathrm{Rh}}\right)=0.0761 \mathrm{~T}_{0}+4.221$ & $\ln \left(F_{\mathrm{Rh}}\right)=0.135 \mathrm{~T}_{10}+4.290$ & $R_{0}^{2}=0.823 \quad R_{5}^{2}=0.722 \quad R_{10}^{2}=0.656$ \\
\hline & $\ln \left(F_{\mathrm{Rh}}\right)=0.132 \mathrm{~T}_{15}+4.352$ & $\ln \left(F_{\mathrm{Rh}}\right)=0.129 \mathrm{~T}_{20}+4.404 \quad \ln \left(F_{\mathrm{Rh}}\right)=0.0902 \mathrm{~T}_{30}+4.827$ & $R_{15}^{2}=0.560 \quad R_{20}^{2}=0.478 \quad R_{20}^{2}=0.220$ \\
\hline & $\ln \left(F_{\mathrm{Rh}}\right)=0.0722 \mathrm{~T}_{40}+5.013$ & $\ln \left(F_{\mathrm{Rh}}\right)=0.0793 \mathrm{~T}_{\mathrm{air}}+4.116$ & $R_{40}^{2}=0.131 \quad R_{\text {air }}^{2}=0.789$ \\
\hline 兴安落叶松-岛状林沼泽 & $\ln \left(F_{\mathrm{Rs}}\right)=0.0713 \mathrm{~T}_{5}+5.0334$ & $\ln \left(F_{\mathrm{Rs}}\right)=0.101 \mathrm{~T}_{10}+4.761$ & $R_{5}^{2}=0.455 \quad R_{10}^{2}=0.497$ \\
\hline \multirow[t]{3}{*}{ Larix gmelinï swamp } & $\ln \left(F_{\mathrm{Rs}}\right)=0.0875 \mathrm{~T}_{15}+4.913$ & $\ln \left(F_{\mathrm{Rs}}\right)=0.0407 \mathrm{~T}_{\text {air }}+4.959$ & $R_{15}^{2}=0.368 \quad R_{\text {air }}^{2}=0.573$ \\
\hline & $\ln \left(F_{\mathrm{Rh}}\right)=-0.0176 \mathrm{~T}_{5}+5.368$ & $\ln \left(F_{\mathrm{Rh}}\right)=0.112 \mathrm{~T}_{10}+4.684$ & $R_{5}^{2}=0.0090 \quad R_{10}^{2}=0.0071$ \\
\hline & $\ln \left(F_{\mathrm{Rh}}\right)=-0.00473 \mathrm{~T}_{15}+5.354$ & $\ln \left(F_{R h}\right)=0.00101 \mathrm{~T}_{\text {air }}+5.301$ & $R_{15}^{2}=0.0049 \quad R_{\text {air }}^{2}=0.0011$ \\
\hline
\end{tabular}

$F$ : 土壤呼吸通量 $\mathrm{CH}_{4}$ flux $T_{X}: X \mathrm{~cm}$ 深度的土壤温度 


\section{3 讨论}

\section{1 土壤呼吸速率的季节动态}

研究表明生长季观测期内两种湿地岛状林沼泽 土壤呼吸速率高峰区都发生在夏季, 呈现出强排放 现象。由图 2 可知, 春季温度逐渐上升、土壤融冻、 植物萌发, 根系呼吸和土壤微生物活性逐渐加强, 使 得呼吸速率呈现逐渐升高变化趋势; 夏季植物生长 达到旺季, 水热条件逐渐达到最佳状态, 植物根系呼 吸与土壤微生物活动强烈, 使得呼吸速率达到最高 水平, 与大多数研究一致 ${ }^{[11,19-22]}$ 。而 8 月下旬后温 度降低, 降水减少, 植物生长日趋衰退, 微生物活动 日趋减弱,致使土壤呼吸速率呈现逐渐下降的趋势。

3.2 生长季沼泽土壤呼吸通量对比

根据观测期实测的寒温带白桦和兴安落叶松岛 状林沼泽土壤呼吸速率数据, 通过分时段计算, 根据 生长季每月上、中、下旬土壤呼吸速率值, 计算各月 份的速率并加和得到土壤呼吸速率在生长季的排放 总量。生长季 (6-10 月) 白华岛状林沼泽 $R_{S}$ 通量为 $12.64 \mathrm{t} / \mathrm{hm}^{2}, R_{H}$ 通量为 $9.89 \mathrm{t} / \mathrm{hm}^{2}, R_{A}$ 通量为 $2.75 \mathrm{t} /$ $\mathrm{hm}^{2}, R_{H}$ 和 $R_{A}$ 分别占土壤呼吸通量的 $81.5 \%$ 和 $18.5 \%$; 落叶松岛状林沼泽 $R_{S}$ 通量为 $10.61 \mathrm{t} / \mathrm{hm}^{2}$, $R_{H}$ 通量为 $7.32 \mathrm{t} / \mathrm{hm}^{2}, R_{A}$ 通量为 $3.29 \mathrm{t} / \mathrm{hm}^{2}, R_{H}$ 和 $R_{A}$ 分别占土壤呼吸通量的 $69.0 \% 、 31.0 \%$ 。由此可得: 两种类型沼泽林土壤微生物呼吸贡献率较高, 明显 高于根系呼吸速率; 当生长季白桦岛状林沼泽土壤 呼吸 $\left(R_{\mathrm{S}}\right)$ 总速率高于落叶松沼泽 $2.03 \mathrm{t} / \mathrm{hm}^{2}$ 情况 下, 土壤异养呼吸 $\left(R_{H}\right)$ 高于落叶松沼泽 $2.57 \mathrm{t} / \mathrm{hm}^{2}$, 而植物根系自养呼吸呼吸 $\left(R_{A}\right)$ 排放量低于落叶松沼 泽 $0.54 \mathrm{t} / \mathrm{hm}^{2}$ 。这表明白桦沼泽土壤微生物呼吸贡 献量高于兴安落叶松沼泽, 而白桦沼泽生态系统根 系呼吸贡献量低于兴安落叶松沼泽。得到这种结果 的原因:一方面调落物作为不同生态系统有机碳主 要来源, 针叶林与阔叶林的碳分配格局, 调落物化学 成分、数量和养分的差异性引起碳排放差异 ${ }^{[23-24]}$, 因 而造成土壤碳转化率的差异, 另一方面调落物分解 速率与土壤水分、地表温度正相关 ${ }^{[25]}$, 白桦沼泽各 土壤层温度均高于落叶松沼泽, 土壤呼吸与 $0-40$ $\mathrm{cm}$ 土壤温度都呈显著相关, 表明微生物活性空间范 围较大, 而落叶松岛状林沼泽土层较薄, 深层土壤温 度较低, 永冻层较高, 因而土壤呼吸速率仅表现为与
5-15 cm 土壤温度呈显著相关, 其微生物活性空间 较小, 贡献率较小。落叶松沼泽根系自养呼吸量较 高于白桦沼泽,可能是其根系分布更贴近地表, 而浅 层土壤温度高于深层土壤温度, 更有利于活根组织 及其根际真菌呼吸。

与其他相关研究相比: 本实验土壤呼吸排放量 低于与本研究生长季降水量接近的小兴安岭 (302.9 $\mathrm{mm})$ 森林湿地白华沼泽 $\left(18.53 \mathrm{t} \mathrm{hm}^{-2} \mathrm{a}^{-1}\right)^{[19]}$ 和落叶 松沼泽(17.49 $\left.\mathrm{t} \mathrm{hm}^{-2} \mathrm{a}^{-1}\right)^{[20]}$, 高于内蒙古大兴安岭 北部兴安落叶松林 $\left(7.06 \mathrm{t} \mathrm{hm}^{-2} \mathrm{a}^{-1}\right)^{[26]}$ 和帽儿山地 区硬阔叶林 $\left(8.92 \mathrm{t} \mathrm{hm}^{-2} \mathrm{a}^{-1}\right)$ 、杨华林 $\left(8.12 \mathrm{t} \mathrm{hm}^{-2}\right.$ $\left.\mathrm{a}^{-1}\right)$ 、落叶松林 $\left(4.51 \mathrm{t} \mathrm{hm}^{-2} \mathrm{a}^{-1}\right)^{[27]}$ 。由此可得: 岛 状林沼泽土壤呼吸速率低于森林湿地, 而高于相关 森林类型, 同时, 阔叶林高于落叶林林型。本研究土 壤呼吸速率低于我国三江平原永久水淹的苔草沼泽 和季节性水淹的小叶章草甸 $\left(19.7-25.7 \mathrm{t} \mathrm{hm}^{-2}\right.$ $\left.\mathrm{a}^{-1}\right)^{[28-29]}$; 接近与北美温带草丛泥炭湿地和冲积平 原湿地 (4.7-13.2 $\left.\mathrm{t} \mathrm{hm}^{-2} \mathrm{a}^{-1}\right)^{[30-31]}$ 、欧洲高纬度北方 泥炭地( 3.4-11.9 $\left.\mathrm{t} \mathrm{hm}^{-2} \mathrm{a}^{-1}\right)^{[32-34]}$ 。由此可得: 寒温 带岛状林沼泽生长季土壤呼吸速率相当于 $\mathrm{CO}_{2}$ 中排 放源, 速率值介于森林湿地和森林林型之间, 白桦岛 状林沼泽土壤呼吸速率高于落叶松沼泽; 微生物的 异养呼吸速率处于主导地位占呼吸速率总量的 $68.0 \%-84.4 \%$, 而根系自养呼吸速率较小, 占呼吸 速率总量的 $15.6 \%-32.0 \%$ 。

\section{3 环境因子对 $\mathrm{CO}_{2}$ 排放通量的影响}

由于气温影响植物的光合作用, 土壤温度则对 土壤微生物活性和植物根呼吸酶的活性有重要影 响 ${ }^{[35-36]}$, 生长季土壤呼吸速率高峰区与高温期具有 较强的一致性, 且其呼吸速率主要集中在夏季。生 长季白桦岛状林沼泽土壤呼吸速率与各层温度相关 性都极显著,兴安落叶松沼泽与 5-15 $\mathrm{cm}$ 土壤温度 存在极显著相关性, 这与现有研究结论基本一 致 ${ }^{[11,28-29,37]}$, 说明细菌对土壤浅层有机质的分解作 用对大兴安岭沼泽生态系统呼吸具有较大贡献, 5 $-15 \mathrm{~cm}$ 土壤温度可能成为其土壤呼吸最佳预测因 子之一。

由于在有氧条件下有机物质具有更高的分解速 率, 土壤呼吸速率对潜水位的变化反映很敏感, 随着 潜水位的降低而增加 ${ }^{[38-39]}$, 水位较高时土壤呼吸速 率受到限制。但本研究观测期内土壤水位保持在较 
低水平,生长季大多观测期内都在 $-40 \mathrm{~cm}$ 以下, 土 壤含水率春季较高, 白桦和落叶松岛状林沼泽土壤 含水率与土壤呼吸相关性分别为 $0.242 、 0.373$, 均未 达到显著水平, 所以不能成为土壤呼吸的限定因子; 但不同林型间比较, 土壤含水率较高的白桦沼泽土 壤呼吸速率高于落叶松沼泽。

此外, 土壤物理和化学性质是影响 $R_{S}$ 的重要因 素 ${ }^{[27]}$ 。在温度和土壤水分相对稳定的情况下, SOC 含量是决定 $R_{S}$ 的重要因子 ${ }^{[28-29]}$ 。本研究白桦岛状 林沼泽土壤 SOC 含量高于兴安落叶松岛状林沼泽, 其呼吸速率也高于兴安落叶松岛状林沼泽。其他研 究也表明, 土壤呼吸 $\left(R_{S}\right)$ 、微生物的异养呼吸 $\left(R_{H}\right)$ 和根系的自养呼吸 $\left(R_{A}\right)$ 与土壤 $\mathrm{A}_{0}$ 和 $\mathrm{A}$ 层的 $\mathrm{SOC}$ 含 量呈显著正相关, 这两个土壤层是调落物和腐殖质 层, 土壤微生物活动大多在这两层, 因此相对较高的 土壤温度势必有利于植物根系呼吸酶的活性和微生 物的分解 ${ }^{[40-41]}$, 但其相关程度因土层而异 ${ }^{[42-45]}$ 。当 硬阔叶林的 SOC 变化范围为 $52.63-66.29 \mathrm{~g} / \mathrm{kg}$ 高 于落叶松 42.15-49.15 g/ $\mathrm{kg}^{[27]}$, 其阔叶林土壤呼吸 速率 $892 \mathrm{gm}^{-2} \mathrm{a}^{-1}$ 高于落叶松林 $451 \mathrm{gm}^{-2} \mathrm{a}^{-1}$, 与本研 究所得结果一致。由此可得到土壤呼吸速率与土壤 SOC 含量相关性明显, 土壤呼吸速率随着 SOC 含量 的增加而增加。此外,实验样地草本生物量、盖度指 标也与土壤呼吸速率大小保持一致, 草本生物量大, 盖度高的其土壤呼吸速率也较大。

致谢:感谢大兴安岭地区农林科学院嫩江源森林生 态定位站同仁对实验的支持。

\section{References:}

[ 1 ] Gates D M. Climate Change and Its Biological Consequences. Massachusetts; Sinauer Associates Inc, 1993.

[2] Tang LZ, Ge XM, Wu L, Ting Y, Wei Y. Partitioning of autotrophic and heterotrophic soil respiration in southern type poplar plantations. Acta Ecologica Sinica, 2012, 32 ( 22) 7000-7008.

[ 3 ] David Risk, Lisakellman Hugo Beltrami Carbon dioxide in soil profile. http://envscilab.stfx.ca./environlab/research3.htm.

[ 4 ] Dixon R K, Solomon A M, Brown S, Houghton R A, Trexier M C, Wisniewski I. Carbon pools and flux of global forest ecosystem. Science, 1994, 263(5144) : 185- 190.

[ 5 ] Law B E, Ryan M G, Anthony P M. Seasonal and annual respiration of a Ponderosa pine ecosystem. Global Chang Biology,
$1999,5(2): 169-182$.

[ 6 ] Singh J S, Gupta S R. Plant decomposition and soil respiration in terrestrial ecosystems. The Botanical Review, 1977, 43 (4): 449-528.

[ 7 ] Gorham E. Northern peatlands: role in the carbon cycle and probable responses to climatic warming. Ecological Applications, 1991,1(2) : 182- 195

[ 8 ] Brix H. Gas exchange through the soil-atmosphere in terphase and through dead culms of Phragmites australis in a constructed reed bed receiving domestic sewage. Water Research, 1990,24(2): 259-266.

[ 9 ] Song C C, Wang Y Y, Wang Y S, Zhao Z C. Character of the Greenhouse Gas Emission in the Freshwater Mire under Human Activities, Scientia Geographica Sinica, 2006,26(1) : 82-86.

[10] Bubier J L, Gaytri B, Moore T R, Roulet N T, Lafleur P M Spatial and Temporal Variability in Growing-season net ecosystem carbon dioxide exchange at a large peatland in Ontario, Canada. Ecosystem, 2003 , 6(1) : 353-367.

[11] Yang J S, Li J S, Wang J D, Yu J B, Sun Z G, Li X H Emissions of $\mathrm{CH}_{4}$ and $\mathrm{N}_{2} \mathrm{O}$ from a wetland in the Sanjiang Plain. Journal of Plant Ecology. 2006,30(3) : 432-440.

[12] Hao Q J, Wang YS, Song CC, Wang YY, Wang M X. Primary study on $\mathrm{CO}_{2}$ and $\mathrm{CH}_{4}$ emissions from wetland soils in the Sanjiang plain.. Journal of Agro-Environment Science, 2004, 23 (5) : 846-851.

[13] Wang D X, Lü X G, Ding W X, Cai Z C, Wang Y Y Comparison of methane emission from marsh and paddy field in Sanjiang Plain. Scientia Geographica Sinica, 2002, 22 (4): 500-503.

[14] Wang D X, Song C C, Wang Y S, Wang Y Y, Yan B X, Zhao Z C. Carbon dioxide flux from peat mire in Ruoergai plateau. Ecology and Environment, 2005,14(6) :880-883.

[15] Hanson P J, Edwards N T, Garten C T, Andrews J A. Separating root and soil microbial contributions to soil respiration: a review of methods and observations.. Biogeochemistry, 2000,48 (1): 115-146.

[16] Cheng S Y, Zhang X Z. A review on differential methods for root and soil microbial contributions to total soil respiration. Earth Science Progress, 2003, 18(4) : 597-602.

[17] Wang C K, Bond-Lamberty B, Gower S T. Soil surface $\mathrm{CO}_{2}$ flux in a boreal black spruce fire chronosequence. Journal of Geop hysicalR esearch, 2002, 107(D3): WFX5-1-WFX5-8.

[18] Wang Y S. Chamber methods for measuring carbon exchange. // Chen PQ ed. Carbon Circling in Earth System. Science Press, Beijing, 2004,130-145. (in Chinese),

[19] Mu C C, Shi LY, Sun X X. Fluxes and controls of $\mathrm{CO}_{2}, \mathrm{CH}_{4}$ and $\mathrm{N}_{2} \mathrm{O}$ in amarsh wetland of Xiaoxing' an mountains, northeastern China. Chinese Journal of Plant Ecology. 2009,33(3) : 617-623.

[20] Mu C C, Cheng W, Sun X X, Wu Y X. Seasonal variation of 
emission fluxes of $\mathrm{CO}_{2}, \mathrm{~N}_{2} \mathrm{O}$ and $\mathrm{CH}_{4}$ from Larix gemlinii Swamps Soils in Xiaoxing'an Mountains of China. Scientia Silvae Sinicae, 2010, 46(7):7-15

[21] Mu C C, Liu X, Sun X X, Shi L Y, Feng D J, Cheng W. Emissions of $\mathrm{CO}_{2}, \mathrm{CH}_{4}$ and $\mathrm{N}_{2} \mathrm{O}$ from broadleaned forested swamp soils in XiaoXing' An Mountains.. Acta ecologica Sinica, 2010b,30 (17) 4598-4608

[22] Song C C, Yan B X, Wang Y S, Wang Y Y, Lou Y J, Zhao Z C. Fluxes of carbon dioxide and methane from swamp and impact factors in Sanjiang Plain, China. Chinese Science Bulletin.2003, 48(24): 2749-2753.

[23] Liikanen A, Huttunen J T, Karjalainen S M, Heikkinen K, Vaisanen T S, Nykanen H, Martikainen P J. Temporal and seasonal changes in greenhouse gas emissions from a constructed wetland purifying peat mining runoff waters. Ecological Engineering, 2006, 26(3): 241-251.

[24] Raich J W, Tufekcioglu A. Vegetation and soil respiration: Correlations and controls. Biogeochemistry, 2000, 48 ( 1 ): 71-90.

[25] Landsberg J J, Waring R H. A generalised model of forest productivity using simplified concepts of radiation-use efficiency, carbon balance and partitioning. Forest Ecology and Management, 1997,9593) : 209-228.

[26] Zhang H D, Zhou M, Zhao P W, Bao Q C, Hai L. Soil respiration of larix gmelinii forest in cool temperate zone. Scientia Silvae Sinicae, 2008, 44(9) :142-145.

[27] Yang J Y, Wang C K. Soil carbon storage and flux of temperate forest ecosystems in northeastern China. Acta ecologica Sinica, 2005,25 (11): $2875-2882$.

[28] Song C C, Wang Y S, Wang Y Y, Zhao Z C. Emission of $\mathrm{CO}_{2}$, $\mathrm{CH}_{4}$ and $\mathrm{N}_{2} \mathrm{O}$ from freshwater marsh during freeze-thaw period in Northeast of China. Atmospheric Environment, 2006, 40 ( 35 ): $6879-6885$

[29] Song C C, Yan B X, Wang Y S, Wang Y Y, Lou Y J, Zhao Z C. Fluxes of carbon dioxide and methane from swamp and impact factors in Sanjiang Plain, China. Chinese Science Bulletin, $2003,48(24):$ 2473-2477. ( in Chinese)

[30] Roehm C L, Roulet N T. Seasonal contribution of $\mathrm{CO}_{2}$ fluxes in the annual $\mathrm{C}$ budget of a northern bog. Global Biogeochemical Cycles, 2003,17( 1) : 1029-1038.

[31] Wickland K P, Striegl R G. Mast M A, Clow D W. Carbon gas exchange at a southern Rocky Mountain wetland, 1996- 1998. Global Biogeochemical Cycles, 2001, 15(2) :321-335

[32 ] Alm J, Schulman L, Walden J, Nykänen H, Martikainen P J, Silvola J. Carbon balance of a boreal bog during a year with an exceptionally dry summer. Ecology, 1999, 80(1) :161- 174.

[33] Waddington J M, Roulet N T. Carbon balance of a boreal patterned peatland. Global Change Biology, 2000, 6( 1) :87-97.

[34] Heikkinen J E P, Virtanen T, Huttunen J T, Elaskov V,
Martikainen P J. Carbon balance in East European tundra. Global Biogeochemical Cycles, 2004, 18(1):1-14.

[35] Andrews J A, Matamala R, Westover KM, Schlesinger W H. Temperature effects on the diversity of soil heterotrophs and the $\delta 13 \mathrm{C}$ of soil respired $\mathrm{CO}_{2}$. Soil Biology and Biochemistry, 2000, 32 ( 5 ) :699- 706

[36] Zogg G P, Zap D R, Ringelberg D B, MacDonald N W, Pregitzer K S, White D C. Compositional and functional shifts in microbial communities due to soil warming. Soil Science Society of America Journal, 1997, 61(2) :475-481

[37] Song C C, Wang Y S, Wang Y Y, Zhao Z C. Emission of $\mathrm{CO}_{2}$, $\mathrm{CH}_{4}$ and $\mathrm{N}_{2} \mathrm{O}$ from freshwater marsh during freeze-thaw period in Northeast of China. Atmospheric Environment, 2006, 40(36) : 6879-6885

[38] Bubier J L, Bhatia G, Moore T R, Roulet N T, Lafleur P M. Spatial and temporal variability in growing-season net ecosystem carbon dioxide exchange at a large peatland in Ontario, Canada. Ecosystems, 2003, 6(4) : 353-367.

[39] Silvola J, Alm J, Ahtholm U, Nykanen H, Martikainen P J. CO fluxes from peat in boreal mires under varying temperature and moisture conditions. Journal of Ecology, 1996, 84(2) : 219-228.

[40] Wen D Z, Wei P, Kong G H, Ye W H. Production and turnover rate of fine roots in two lower subtropical forest sites at Dinghushan. Chinese Journal of Plant Ecology, 1999, 23 (4) : 361-369.

[41] Zhang X Q, W u K H, Fine root production and turnover for forest ecosystems. Scientia Silvae Sinica, 2001, 37 ( 3 ): 126- 138 .

[42] Geng Y B, Zhang S, Dong Y S. The content of soil organic carbon and total nitrogen and correl activity between their content and fluxes of $\mathrm{CO}_{2}, \mathrm{~N}_{2} \mathrm{O}$ and $\mathrm{CH}_{4}$ in Xilin River Basin Steppe. Acta Geographica Sinica, 2001, 56 (1) : 44-53.

[43] Li M F, Dong Y S, Geng Y B, Qi Y C. A nalyses of the correlation between the fluxes of $\mathrm{CO}_{2}$ and the distribution of $\mathrm{C}$ $\& N$ in Grassland Soils. Environmental Science, 2004, 25 (2) : 7-11.

[44] Xu M, Qi Y. Soil-surface $\mathrm{CO}_{2}$ efflux and its spatial and temporal variations in a young ponderosa pine plantation in northern California. Global Change Biology, 2001, 7(6) : 667-677.

[45] Kang S Y, Doh S, Lee D. Topographic and climatic controls on soil respiration in six temperate mixed-hardwood forest slopes, Korea. Global Change Biology, 2003, 9(10): 1427-1437.

\section{参考文献:}

［2］唐罗忠, 葛晓敏, 吴麟, 田野,魏勇. 南方型杨树人工林土壤呼 吸及其组分分析. 生态学报,2012,32(22) 7000-7008.

[9] 宋长春, 王毅勇, 王跃思, 赵志春. 人类活动影响下淡水沼泽 湿地温室气体排放变化. 地理科学, 2006,26(1) : 82-86.

［12］郝庆菊, 王跃思, 宋长春, 王毅勇, 王明星. 三江平原湿地土 
壤 $\mathrm{CO}_{2}$ 和 $\mathrm{CH}_{4}$ 排放的初步研究. 农业环境科学学报, 2004,23 (5) : 846-851.

[13] 王德宣, 吕宪国, 丁维新, 蔡祖聪, 王毅勇. 三江平原沼泽湿地 与稻田 $\mathrm{CH}_{4}$ 排放对比研究. 地理科学, 2002, 22(4) : 500-503.

［14］王德宣, 宋长春, 王跃思, 王毅勇, 阎百兴, 赵志春. 若尔盖 高原泥炭沼泽湿地 $\mathrm{CO}_{2}$ 呼吸通量特征. 生态环境, 2005,14 (6) : 880-883.

[16] 程慎玉, 张宪洲. 土壤呼吸中根系与微生物呼吸的区分方法与 应用.地球科学进展, 2003, 18(4): 597-602.

[18］王跃思. 碳交换的箱法测定. //陈泮勤, 地球系统碳循环, 北 京: 科学出版社, 2004,130-145.

[19］牟长城, 石兰英, 孙晓新.小兴安岭典型草丛沼泽湿地 $\mathrm{CO}_{2}$ 、 $\mathrm{CH}_{4}$ 和 $\mathrm{N}_{2} \mathrm{O}$ 的排放动态及其影响因素.植物生态学报, 2009, $33(3): 617-623$.

[20] 牟长城, 程伟, 孙晓新, 吴云霞. 小兴安岭落叶松沼泽林土壤
$\mathrm{CO}_{2}, \mathrm{~N}_{2} \mathrm{O}$ 和 $\mathrm{CH}_{4}$ 的排放规律..林业科学, 2010,46(7) :7- 15

[21］牟长城, 刘霞, 孙晓新, 冯登军, 石兰英.小兴安岭阔叶林沼泽 土壤 $\mathrm{CO}_{2} 、 \mathrm{CH}_{4}$ 和 $\mathrm{N}_{2} \mathrm{O}$ 排放规律及其影响因子, 生态学报, 2010, 30 (17) :4598-4608.

[22］宋长春, 阎百兴,王跃思, 王毅勇, 娄彦景, 赵志春. 三江平原 沼泽湿地 $\mathrm{CO}_{2}$ 和 $\mathrm{CH}_{4}$ 通量及影响因子..科学通报, 2003,48 (23) :2473-2477。

[26] 张慧东, 周梅, 赵鹏武,包青春, 海龙. 寒温带兴安落叶松林土 壤呼吸特征.林业科学, 2008,44(9): 142-145

[27] 杨金艳, 王传宽. 东北东部森林生态系统土壤碳咜量和碳通 量.生态学报, 2005, 25(11)2875-2882.

[40］温达志,魏平, 孔国辉,等. 鼎湖山南亚热带森林细根生产力与 周转. 植物生态学报, 1999, 23 (4) : 361-369.

[41] 张小全, 吴可红. 森林细根生产和周转研究. 林业科学, 2001, 37 (3) : 126-138. 